Referencia para citar este artículo: Ordaz-Mejía, D. A. \& Osorio-García, M. (2018). Significados del viaje turístico en jóvenes millenials. Caso de estudio: Texcoco, México. Revista Latinoamericana de Ciencias Sociales, Niñez y Juventud, 16(2), 897-912. doi:https://doi.org/10.11600/1692715x.16217

\title{
Significados del viaje turístico en jóvenes millenials. Caso de estudio: Texcoco, México*
}

\author{
Denice ANEL Ordaz-MEJía ** \\ Egresada Universidad Autónoma del Estado de México, México. \\ MARIBEL OSORIO-GARCÍA*** \\ Profesora Universidad Autónoma del Estado de México, México.
}

\section{Artículo recibido en noviembre 17 de 2017; artículo aceptado en enero 15 de 2018 (Eds.)}

- Resumen (analítico): desde un paradigma interpretativo, realizamos un estudio sobre las creencias y valores que orientan los viajes turísticos de los y las gentes jóvenes millenials para establecer su tipificación turística. Con este objetivo, adelantamos una investigación cualitativa con jóvenes estudiantes de la Universidad Autónoma del Estado de México, en la región de Texcoco de la zona metropolitana de la ciudad de México. Sustentamos el planteamiento teórico en Schütz y Lalive, considerando el ethos como categoría base. Las técnicas que utilizamos fueron la entrevista - aplicada a 29 jóvenes de manera interactiva-, y el análisis del discurso, desagregado en las dimensiones temporal, social y espacial. Los hallazgos nos permitieron configurar dos ethos: el tradicional y el liberador, cuya diferenciación radica en los anclajes socioculturales que tiene la gente joven a partir de sus creencias y valores.

Palabras clave: joven, viaje, turismo, valores sociales (Tesauro de Ciencias Sociales de la Unesco).

\section{Meaning of travel for millennial young people in Mexico. Case study: Texcoco, Mexico}

- Abstract (analytical): Using an interpretative approach, this research involved the study of the beliefs, values and models that shape the experience of travelling for millennial young people in Mexico was carried out to classify what types of tourists are visiting Texcoco. A qualitative study was carried out by a group of young university students from the Universidad Autónoma del Estado de México, in the Texcoco region of the metropolitan area of Mexico City. The theoretical approach is based on the work of Schütz and Lalive, taking ethos as a base category. The methodological technique used was the interview, applying a discourse analysis to the meta-texts obtained in their temporal, social and spatial dimensions. The findings facilitated the configuration of two ethos: the

Este artículo de investigación científica y tecnológica se deriva de la investigación denominada «Valores e imaginarios de los jóvenes universitarios en torno al viaje turístico. Caso de estudio: Universidad Autónoma del Estado de México, campus Texcoco», presentada por la primera autora y asesorada por la segunda para optar al título de Maestra en Estudios Turísticos, Facultad de Turismo y Gastronomía, Universidad Autónoma del Estado de México, México, 2017, realizada entre agosto de 2015 y julio de 2017 (Clave de Conacyt: CVU 702650). El artículo se ubica en las Ciencias Sociales, en el área y subárea de conocimiento de la Sociología.

** Licenciada en Turismo por la Universidad Autónoma del Estado de México (UAEM), campus Texcoco. Becaria de la Maestría en Estudios Turísticos de la UAEM, programa inscrito en el Padrón Nacional de Posgrados de Calidad (PNPC) del Consejo Nacional de Ciencia y Tecnología (Conacyt) de México. Orcid: 0000-0002-2871-3868 Correo electrónico: denice091287@hotmail.com

*** $\quad$ Autora por correspondencia. Licenciada en Turismo de la UAEM, Magíster en Planeación Urbana y Regional de la UAEM, Doctora en Ciencias Sociales y Políticas (especialidad en Sociología), de la Universidad Iberoamericana (México), profesora-investigadora de tiempo completo de la UAEM. Profesora invitada al Doctorado en Gestión Turística de la Universidad del Occidente (México) y al Doctorado en Geografía de la Universidad Nacional Autónoma de México (Unam), inscritos en el PNPC del Conacyt. Orcid: 0000-0002-3336-6231; Indice H5: 18. Correo electrónico: maribelosorio2@gmail.com 
traditional and the liberator, with the differentiation lying in the sociocultural anchors that young people have from their beliefs and values.

Key words: Young people, travel, tourism, social values (Social Science Unesco Thesaurus).

\section{Significados de viagem turística para os jovens millenials do México. Estudo de caso: Texcoco, México}

- Resumo (analítico): desde um paradigma interpretativo, um estudo foi realizado sobre as crenças e valores que orientam as viagens turísticas dos jovens millenials para estabelecer sua tipificação turística. Com esse objetivo, a pesquisa qualitativa foi realizada com jovens estudantes da Universidade Autônoma do Estado do México, na Texcoco, na região metropolitana da Cidade do México. A abordagem teórica é baseada em Schütz e Lalive, considerando o ethos como uma categoria de base. As técnicas utilizadas foram a entrevista, aplicada a 29 jovens de forma interativa e a análise do discurso, desagregada nas dimensões temporal, social e espacial. As descobertas permitem configurar dois ethos: o tradicional e o libertador, cuja diferenciação reside nas âncoras socioculturais que os jovens têm de suas crenças e valores.

Palavras-chave: jovem, viagem, turismo, valores sociais (Thesaurus de Ciências Sociais da Unesco).

-1. Introducción. -2. Encuadre teórico-conceptual. -3. Ubicación de los sujetos de estudio. -4. Modelo metodológico. -5. Hallazgos. -6. Reflexiones finales. -Lista de referencias.

\section{Introducción}

Las personas nacidas entre los años 1980 y 2000, ahora conocidos como «millenials» o generación Y, se encuentran en la etapa de juventud, con un estilo de vida que caracteriza y diferencia esta generación de sus predecesoras (Jacomo, 2016), la generación de los baby boomers (nacidos entre 1943 y 1960) y la generación X (nacidos entre 1960 y 1980) (Dwyer, Edwards, Mistilis, Roman, Scott, \& Cooper, 2008). Según Jacomo (2016), Lundy (2015), Geifman (2014), The Council of Economic Advisers (2014) y Dwyer et al. (2008), los millenials se caracterizan por ser nativos y nativas digitales, conectados permanentemente a sus redes sociales en tiempo real. Son individuos que trabajan para vivir y no a la inversa; buscan nuevas formas de entretenimiento, propósitos gratificantes y significados espirituales. Tienen una forma peculiar de practicar el turismo: se estima que el $90 \%$ de las personas millenials planifican sus viajes usando recursos tecnológicos (Hostetur, 2016); dedican $20 \%$ de sus ingresos económicos para viajar, mostrando nuevos hábitos de compra (Reporte Lobby, 2017); gustan de experimentar la vida diaria y la cultura de los lugares visitados (Dwyer et al., 2008); y sienten preocupación por el cuidado del medio ambiente (Miranda \& Yanchaguano, 2017).

Así también, se ha registrado que las personas jóvenes demandan rutas nuevas (He, Yang, \& Guan, 2014), prefieren actividades de aventura y visitas a lugares donde se aprecia la naturaleza (Posso \& Cardona, 2016), ansían adquirir nuevas experiencias o conocimientos (Korstanje, 2010) y utilizan permanentemente la Internet y las redes sociales durante sus viajes (Biz, Neves, \& Bettoni, 2014; Hu, Zhao, \& Huang, 2015). Ortega (en Unwto, 2012) menciona que el turismo de jóvenes, en mayor medida que otros segmentos, es consciente de los problemas sociales y ambientales de los destinos turísticos, y potencia el diálogo y el entendimiento mutuo entre las culturas. Los individuos millenials consideran los viajes como una parte esencial de su vida cotidiana en lugar de un breve escape de la realidad (Matteucci, en Unwto, 2012).

Es claro que los diferentes contextos y estilos de vida de los sujetos jóvenes les influyen en su comportamiento (Bermúdez, 2008). La juventud responde a una construcción de identidad como grupo social, capaz de crearse a sí misma en relación con los otros, y construir signos, símbolos y toda una visión del mundo en una construcción social determinada (Cardoso, 2005). El turismo se 
configura como una expresión de esa construcción social (Binkhorst, 2008; Del Barrio, López, \& Frías, 2012; Guimarães, 2012; He et al., 2014; Korstanje, 2010; Marinao, Torres, \& Chasco, 2014; Orgaz \& López, 2015), en la que se vierte el sentido del viaje, delineado por unos valores y significados que manifiestan distintas fantasías y deseos. Esto se puede observar en el caso de los spring breakers en México o los schoolies en Australia, quienes expresan en forma extrema su vivencia del viaje mediante el abuso de sustancias tóxicas y una actividad sexual de alto riesgo (De Salazar, 2003; Monterrubio, 2013; Olivares, 2009).

Para el caso de México, gracias a distintas investigaciones (Barragán et al., 2014; Consejo Nacional de Población, 2010; Lehmann, 2015; Pérez-Rodríguez, 2012; Sánchez, 2014; Sima \& Perales, 2015), podemos decir que se tiene conocimiento sobre el sector juvenil; sin embargo, en general se observa una carencia de estudios sobre el turismo de las personas jóvenes millenials, y en particular, una carencia de investigaciones de carácter social que estudien el significado del viaje turístico para este grupo. Reconocer este significado resulta importante para una sociedad en la que el turismo doméstico constituye el 80\% de su actividad turística nacional (Gobierno de la República de México, 2013), y en la que los sujetos millenials definirán el comportamiento turístico del país en los próximos años, no solo por su consumo, sino a partir de sus pautas de comportamiento sociocultural.

En el contexto de la zona metropolitana de la ciudad de México (ZMCM) (Cruz, 2000), se considera que el perfil de los individuos jóvenes millenials se acerca mayormente al del sujeto joven «global», dada su condición cosmopolita; sin embargo, como nación multicultural y «en desarrollo», se conjugan también las tradiciones y valores ancestrales con el estilo de vida urbano y los valores modernos (Pérez-Rodríguez, 2012; Valdés, 2013). De hecho, la Encuesta Mundial de Valores (http:// www.worldvaluessurvey.org/wvs.jsp) confirma que los valores de la sociedad mexicana se perfilan entre la tradición y la autoexpresión (Valdés, 2013), en donde la tradición otorga preponderancia: a los lazos entre padres/madres e hijos/hijas, a la deferencia a la autoridad, y a los valores tradicionales de la familia; y la auto-expresión otorga importancia al bienestar subjetivo, a la auto-expresión, y a la calidad de vida.

La constitución de la identidad particular de la gente joven se expresa en distintos ámbitos, entre ellos, el turismo. Bermúdez (2008) plantea que no hay una única manera de ser joven, sino diversas maneras, puesto que en las ciudades hay una heterogeneidad de espacios y consumos culturales para construir sus identidades. A partir de esta premisa, se infiere que no existe un sentido único del viaje turístico en los individuos jóvenes, y que la construcción de sus significados está articulada a un conjunto de creencias y valores. Por ello, es de interés en esta investigación llevar a cabo una aproximación a la identificación de los significados del turismo en las personas jóvenes millenials de Texcoco en la ZMCM, con el objetivo de reconocer las creencias, valores y modelos que lo orientan, así como su tipificación turística. Justo por la condición de territorio metropolitano periférico, elegimos Texcoco para estudiar los significados del turismo en su población joven, bajo el supuesto de que dichos sujetos jóvenes conjugan anclajes tradicionales y patrones modernos en su perfil sociocultural.

Con ese propósito, el texto que ofrecemos lo hemos estructurado en cuatro apartados: el primero está referido al encuadre teórico-conceptual que define las categorías de la tipificación y el ethos como conceptos base para el estudio interpretativo; en un segundo apartado describimos el grupo de jóvenes elegido de la ZMCM que fungieron como sujetos de la investigación; en el tercero presentamos el modelo metodológico y las técnicas cualitativas aplicadas; y en el cuarto exponemos los hallazgos de la investigación en sus dimensiones temporal, social y espacial, así como las tipificaciones turísticas en los dos ethos identificados. Cerramos el texto con un apartado de reflexiones finales.

\section{Encuadre teórico-conceptual}

Debido a que las llamadas sociologías de la vida cotidiana son las que han mostrado un interés más decidido por estudiar el sentido y los significados del hacer humano (Lindón, 2000), el encuadre teórico de esta investigación queda circunscrito al ámbito de la investigación subjetiva, específicamente 
en el campo fenomenológico del sentido, tomando como base los planteamientos de Alfred Schütz (1995), Schütz y Luckmann (1973) y Lalive (1990). Como es conocido, Schütz basó su teoría en las ideas de Husserl y Weber inclinando sus estudios hacia la subjetividad humana, ya que para el «... el mundo de la vida cotidiana es (...) la realidad fundamental y eminente del hombre...» (Schütz \& Luckmann, 1973, p. 25).

Schütz (1995) planteó que el mundo social se estructura desde la vida cotidiana donde el ser humano construye significados intersubjetivos. Se entiende por significados los conceptos-códigos que se comparten socialmente y constituyen los aspectos del mundo social que son importantes para los sujetos (Ritzer, 1993, p. 280). En la misma línea, Crespi (1997) conceptualiza el significado como cualquier representación del sí mismo y de la realidad. Las experiencias y conocimientos que se albergan en la conciencia dan sentido y significado diferente a las cosas y saberes, conformando un acervo. En palabras de Berger y Luckmann (1997), «la constitución subjetiva del sentido es el origen de todos los acervos sociales de conocimiento, los depósitos históricos de sentido en que pueden apoyarse las personas nacidas en una sociedad y en épocas particulares» (p. 35). Schütz denominó este acervo como «sentido común», construido por significados colectivamente entendidos en un determinado momento que orientan las formas de interacción socialmente aceptadas y sabidas por los miembros de una comunidad. La transmisión de sentido está determinada por las relaciones intersubjetivas que soportan la estructura social.

El sentido común se compone por la situación biográfica, el acervo social de conocimientos y el contexto geográfico, y se produce mediante la supuesta comprensión de las acciones de los demás. El sentido que una persona asigna a su acción se constituye en una forma típica de conducta, una pauta típica de motivos subyacentes, de actitudes típicas de un tipo de personalidad. Cabe señalar que Schütz extrae de Weber la idea de las «tipificaciones», para simplificar la aprehensión conceptual de actos y conductas humanas que ayuden a determinar los tipos de personalidad individual o colectiva en una estructura social. Las tipificaciones se enuncian como un conjunto de reglas y recetas que hacen posibles las relaciones interpersonales, al anticipar la conducta en el mundo de la vida cotidiana (Schütz, 1995).

Para identificar las tipificaciones, retomamos aquí el concepto ethos, desde la perspectiva teórica que establece Lalive (1990), quien lo concibe como el conjunto de creencias, valores, normas y modelos que orientan el comportamiento y constituyen la identidad socio-cultural del individuo. El ethos forma el núcleo de una cultura que caracteriza a una institución, a un grupo o a una sociedad, por lo que es una herramienta teórica pertinente para la identificación de las tipificaciones en el grupo social de los sujetos jóvenes.

Bajo el enfoque fenomenológico, Lalive (1990) propone un modelo operativo de análisis para llevar a cabo la interpretación de los significados personales y hacer observables las tipificaciones en las que se está inmerso. El modelo consiste en analizar la subjetividad inmersa en un relato biográfico. Se parte de la idea de que la vida humana se inserta en un espacio tridimensional, y cada dimensión es interdependiente y no puede distinguirse más que por el artificio del análisis. Dicho marco supone las relaciones más significativas de estas inserciones en la historicidad del sujeto, y orienta la detección de información para la reconstitución del ethos (Reyes \& Osorio, 2015; Vallejo, Osorio, Ramírez, Nava, \& Franco, 2013). El modelo hace posible definir las llaves de interpretación específicas para una investigación y los cruces con las dimensiones temporal, social y espacial, como ejes estructuradores de la vida cotidiana. En la dimensión temporal se distingue la relación del tiempo en la vida del sujeto: la fase del ciclo de vida en la cual se sitúa, así como la inserción del sujeto en la historicidad o en el tiempo de la sociedad. En la dimensión social, se identifica la relación del sujeto consigo mismo (yo-yo) y con los otros, tanto con quienes establece una relación directa (yo-tú/yo-nosotros), como con quienes se diferencia (yo-ellos/nosotros-ellos). En la dimensión espacial se reconocen los espacios personales de la vida cotidiana (casa, escuela, trabajo), así como los espacios externos a ella (la región, el país, el extranjero). 
Aunque no hay acceso directo a las experiencias subjetivas de otros, es posible comprender su naturaleza y contenido mediante las expresiones y la información que proporcionan los sujetos en estudio (Ritzer, 1993), lo que se logra a través de la investigación cualitativa, que es pertinente para explicar los fenómenos que se suscitan dentro de un contexto natural de acuerdo con los significados que tienen para las personas implicadas (Denzin \& Lincoln en Rodríguez, 1999; Hernández, Fernández, \& Baptista, 2014).

\section{Ubicación de los sujetos de estudio}

La ZMCM se localiza en el centro del país y está constituida por 16 delegaciones del Distrito Federal — asentamiento de la ciudad de México-, 58 municipios del Estado de México y 1 municipio del Estado de Hidalgo, concentrando una población estimada de 20 millones de habitantes para el año 2010 (Pérez \& Santos, 2011).

El acelerado crecimiento de la zona — registrado a partir de la década de 1970 - implicó un proceso de conurbación en su entorno con poblaciones que para entonces tenían una condición rural. Tal es el caso de Texcoco, asentamiento de origen prehispánico que conformó una de las más importantes aglomeraciones metropolitanas en el área urbana de la periferia a partir de los años ochenta (Cruz, 2000) (Figura 1).

Figura 1. Mapa de Localización de Texcoco en la Zona Metropolitana de la ciudad de México, México
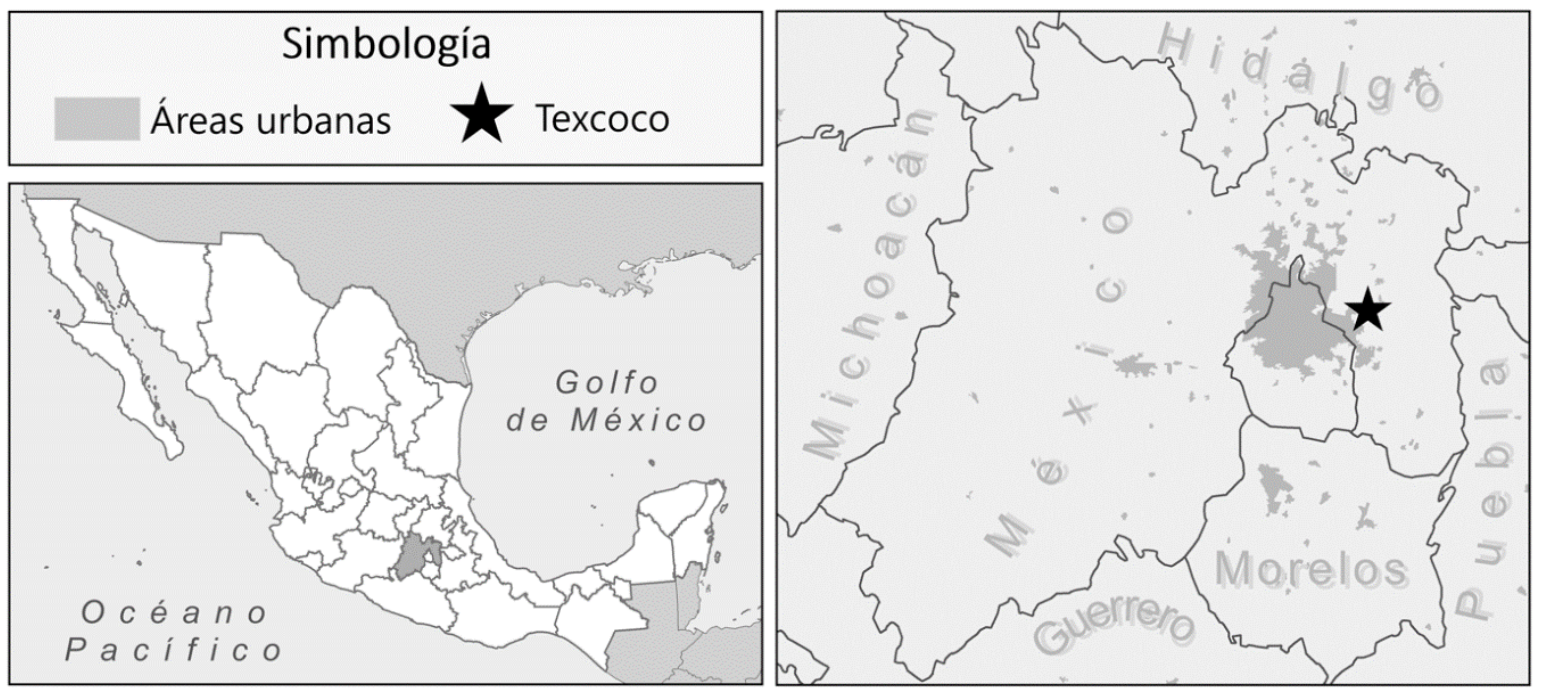

Nota. Elaboración con base en datos del Instituto Nacional de Estadística y Geografía de México.

Para el año 2015 se estimó que la población del municipio de Texcoco de Mora era de 240.749 personas, y continuaba desempeñando un valioso papel en la dinámica de la ZMCM, por ser uno de los municipios más cercanos a la Ciudad de México y el segundo más urbanizado de su región. De acuerdo con la información oficial, para el año 2016 su condición de desarrollo era ligeramente mayor a los niveles promedio del país: $53.1 \%$ de la población municipal tenía un ingreso superior a la línea de bienestar y $57.85 \%$ se encontraba por arriba de la línea de pobreza (H. Ayuntamiento de Texcoco, 2016), en tanto las cifras de la población nacional eran de $49.4 \%$ y 56.4\%, respectivamente (Consejo Nacional de Evaluación de la Política de Desarrollo Social-Coneval, 2017). Lo anterior, en función de que su actividad económica se basa en la industria, el comercio y los servicios (H. Ayuntamiento de Texcoco, 2016). 
Para cubrir los criterios de que fueran jóvenes millenials con un nivel educativo acorde a su edad (educación superior), optamos por llevar a cabo la investigación con estudiantes del Centro Universitario Texcoco de la Universidad Autónoma del Estado de México (CUT), institución pública de reconocido prestigio en México. Según un diagnóstico de la población estudiantil del CUT realizado por Alejo, Mendoza y Rodríguez (2012), 65\% de los individuos encuestados consideran que su ingreso económico se encuentra entre medio y alto, ya que además de cubrir las necesidades básicas como alimentación, vivienda y vestido, tienen acceso a productos y servicios de mayor confort: $77.6 \%$ contaba con computadora, $52.5 \%$ tenía Internet en su casa, $40.9 \%$ tenía automóvil y $44 \%$ poseía tarjeta de crédito. Estas condiciones ubican a este grupo de estudiantes en lo que el Instituto Nacional de Estadística y Geografía en México ha clasificado como clase media (Inegi, 2013), dado el nivel de gasto y los bienes y servicios con los que cuentan. La clase media corresponde al nivel socioeconómico C (López, 2010), estrato que considera a las personas con ingresos o nivel de vida medio. Dicha condición garantiza que estas personas jóvenes tengan capacidad de gasto para servicios de esparcimiento y viajes.

Mediante la información otorgada por la Subdirección Académica del CUT, se conoce que en el periodo escolar 2016-2017 fueron matriculados un total de 3815 alumnos y alumnas: 1679 hombres y 2136 mujeres, distribuidos en las nueve carreras de Licenciatura que se imparten en el plantel: Administración (LAM), Economía (LEC), Ciencias Políticas y Administración Pública (LCP), Contaduría (LCN), Derecho (LDE), Informática Administrativa (LIA), Lenguas (LLE), Ingeniería en Computación (ICO) y Turismo (LTU). Explicamos a continuación la forma en que procedimos para la investigación cualitativa.

\section{Modelo metodológico}

Para la aplicación del modelo de Lalive, recabamos la información de los sujetos de estudio mediante la técnica de la entrevista, obteniendo un relato autobiográfico que transcribimos para crear un meta-texto, el cual fue sometido a un análisis del discurso. Los individuos jóvenes a quienes aplicamos la entrevista eran estudiantes de entre 18 y 25 años, de las nueve licenciaturas del CUT, de los turnos matutino y vespertino, que cursaban entre el primer y noveno semestres de sus respectivas carreras. Llevamos a cabo 29 entrevistas en las instalaciones de la propia Universidad, con la participación voluntaria de los alumnos y las alumnas. Presentamos una ficha técnica de las entrevistas en la tabla 1.

Tabla 1. Individuos Jóvenes Entrevistados en el CUT, México. Ficha técnica

\begin{tabular}{|c|c|}
\hline Rubro & Datos \\
\hline Total de jóvenes & 29 \\
\hline Género & 15 mujeres y 14 hombres \\
\hline Rango de edad & Entre 18 y 25 años \\
\hline Semestres & Entre primero y noveno \\
\hline Lugar de procedencia & 12 de Texcoco \\
& 17 de la ZMCM \\
\hline Ocupación & 21 estudian \\
& 7 estudian y trabajan \\
& 1 estudia y es ama de casa \\
\hline Periodo de aplicación & Noviembre 2016 - Febrero 2017 \\
\hline
\end{tabular}


Como se puede observar, contamos con un número casi equivalente de hombres (14) y mujeres (15); además, aunque la mayoría de los alumnos y alumnas proceden de la propia localidad, también incluimos sujetos provenientes de sitios aledaños (Tepetlaoxtoc, Los Reyes, Chiconcuac, Chimalhuacán, Ecatepec, Ixtapaluca y San Vicente Chicoloapan), todos ellos dentro de la ZMCM.

La llave de interpretación central fue el viaje turístico, con la cual se cruzan las dimensiones temporal, social y espacial en el análisis del discurso y se abstrae la subjetividad implícita en los relatos. Cabe señalar que de acuerdo con Osorio (2007), el viaje turístico se entiende como una forma de disfrute, como una práctica que se lleva a cabo de manera voluntaria y recreativa, como manifestación de ocio. Particularmente, su sentido se establece a partir de distinguir su práctica en el tiempo y el espacio, es decir, es una acción que se realiza durante el tiempo libre — no en el tiempo de trabajo - y en un espacio de vida no cotidiana, para distinguir la diferencia con el entorno habitual.

Adicionalmente consideramos dos llaves de interpretación complementarias para enriquecer el perfil sociocultural de los sujetos: la vida cotidiana - como elemento nodal de la subjetividad-, y la universidad — como campo de las interacciones sociales de los sujetos jóvenes elegidos-. En la Figura 2 presentamos el esquema del modelo interpretativo aplicado.

Figura 2. Modelo interpretativo aplicado para el análisis del discurso

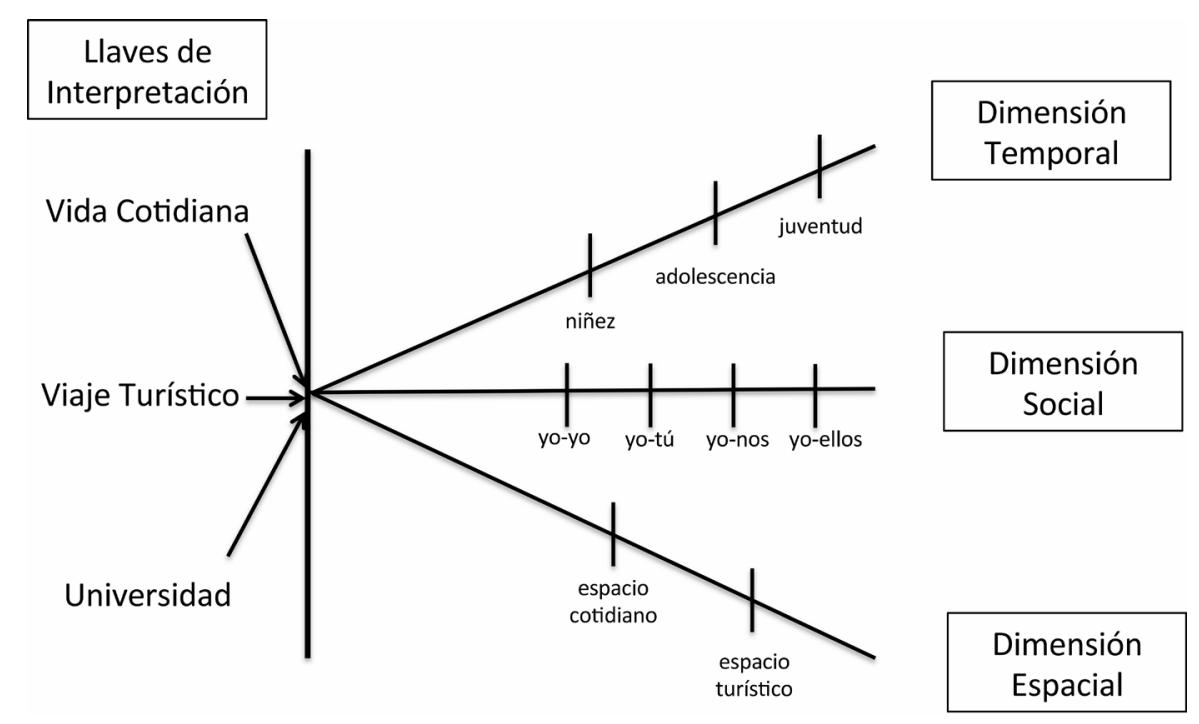

Nota. Elaboración con base en el modelo de Lalive (1990).

En el caso de la dimensión temporal, establecimos tres unidades para analizar el flujo histórico del grupo de estudiantes: la niñez, la adolescencia y la juventud, con el propósito de identificar el sentido y significado que el viaje representó en cada uno de estos estadios. En la dimensión social, analizamos las interacciones del sujeto consigo mismo (yo-yo) y con los otros (yo-tú, yo-nos), tanto quienes forman parte de su núcleo íntimo (la familia y los amigos y amigas), como quienes son ajenos a este (yo-ellos) (compañeros y compañeras, docentes). En la dimensión espacial, consideramos las unidades de análisis del espacio de su vida cotidiana y del espacio turístico, que comprende el espacio de los viajes.

Cabe aclarar que nuestra pretensión en la presente investigación no fue alcanzar generalizaciones - como sucede para el caso de las investigaciones cuantitativas-, sino descripciones del mundo percibido por los agentes sociales, mediante interpretaciones fidedignas sobre los significados del viaje desde la intersubjetividad del individuo (Kvale, 2011). Como se establece para la investigación cualitativa, alcanzamos el punto de saturación en el estudio con las 29 entrevistas aplicadas, al encontrar repeticiones de los significados ya expresados en las unidades de análisis establecidas. 


\section{Hallazgos}

\section{Dimensión temporal}

Por una parte, las personas jóvenes millenials refieren una niñez con pasajes felices y activos, en la que sus familiares cercanos fueron dando sentido a su vida e inyectaron en ellos el aprecio por la familia y valores relacionados con el amor y la solidaridad a través de la convivencia cotidiana con sus padres, madres, hermanas y hermanos. Resaltan que su generación todavía tuvo una socialización vivenciada por la interacción humana comunitaria y directa, antes del cambio de hábitos pasivos e individuales que tienen ahora los niños y las niñas por los medios electrónicos y la Internet. Por otra parte, también hubo relatos que rememoraban la ausencia de sus padres o madres por compromisos laborales o sociales, aludiendo a experiencias de abandono y de maltrato escolar por compañeros y compañeras de clase.

Los recuerdos de los viajes que realizaron los jóvenes y las jóvenes durante su etapa infantil son profundos; incluso para algunos de ellos son los más significativos de su vida, pues propiciaron intensos momentos de convivencia con sus padres, madres y familiares cercanos, en un ambiente de armonía y gozo; familiares que incluso han fallecido: «ese viaje fue en la playa con mi abuelo, fue dos años antes de que falleciera, fue muy importante para mí porque fue su último viaje» (Ent. 2, Comunicación personal, 01 noviembre, 2016).

Sobre la etapa adolescente, estas personas jóvenes enfatizaron los cambios físicos y conductuales que ocurrieron, resaltando sus rebeldías, las primeras experiencias en el consumo de bebidas alcohólicas y el desequilibrio emocional que sufrieron, expresando incluso sus pretensiones autodestructivas: «pues es que muchas veces la loquera tan horrible... luego agarraba un cuchillo y me lo quería enterrar» (Ent. 22, Comunicación personal, 15 febrero, 2017). Manifestaron también que en esta etapa hicieron conciencia de nuevos desafíos para su vida, asumiendo la responsabilidad de seleccionar una trayectoria profesional para forjarse un futuro promisorio y corresponder a los esfuerzos de su familia. En esta etapa se viven las primeras experiencias de emancipación a través de excursiones o salidas de su entorno inmediato, con o sin permiso de la autoridad familiar o escolar, fundamentalmente en busca de breves momentos de diversión.

En la etapa juvenil, las salidas de los individuos entrevistados se diversifican en viajes de diversión, de socialización y de estudio, cambiando su sentido sobre el viaje y dotándolo de, al menos, dos importantes significados: la liberación y el desarrollo personal. Por un lado, se vive una posibilidad de liberación y rompimiento temporal de convencionalismos marcados por sus instituciones y normas sociales, muy arraigadas en su contexto. Los viajes realizados a distintos Estados del país (ver Dimensión Espacial) se tornan en oportunidades para «gozar» de su juventud y desinhibirse, incluso de manera desenfrenada:

(...) era pura fiesta, nos llevaban alcohol ilimitado, los chavos del staff eran muy guapos y echaban fiesta con nosotros, ya sabes te besas con uno y con otro para cotorrear. Pero el viaje fue de pura fiesta y alcoholizarnos... tomé y me divertí mucho... fumamos mucho... mis amigos sí compraban droga como marihuana y coca que allá vendían... (Ent. 16, Comunicación personal, 22 noviembre, 2016).

Por otro lado, el viaje se asume como una opción para alcanzar un mayor desarrollo personal y contribuir al desenvolvimiento social: «(...) tienes que conocer, bueno tienes que desenvolverte, ver hacia otros horizontes y cosas así... es como un libro, sí, está padre, a mí sí me gusta viajar...» (Ent. 12, Comunicación personal, 09 noviembre, 2016). Además de que estas personas jóvenes le atribuyen distintos elementos de estatus por ser «un lujo», condicionado a la posibilidad de contar con «tiempo y dinero» para su realización, por lo que «(...) no siempre se puede...» (Ent. 4, Comunicación personal, 02 noviembre, 2016), así también el desenvolvimiento social se considera en dos sentidos: 
permite un roce social con personas de otros lugares y condiciones socioeconómicas de nivel medio y alto, e ilustra cómo actuar y conducirse en transacciones comerciales y uso de servicios: «(...) 1legas a un restaurante y muchas veces no sabes cómo tratar con las personas, así como ¿qué hago? ¿cómo pido?... me ha ayudado a desenvolverme en cualquier lugar...» (Ent. 29, Comunicación personal, 17 febrero, 2017).

\section{Dimensión social}

Con respecto a la dimensión social, en la relación yo-yo el viaje otorga a los sujetos jóvenes una imagen sobre sí mismos; en la relación yo-otros conforma una socialización centrada en dos núcleos principales: la familia y los compañeros y compañeras de la universidad. Estos individuos jóvenes manifiestan una satisfacción por los estudios que se encuentran realizando y aprecian los viajes como alicientes para su superación, incluso como elementos de su propia formación profesional. Señalan que los viajes les «abren la mente», en el sentido de que les generan procesos de autorreflexión sobre quiénes son y lo que desean hacer en su vida: «Una noche queríamos ver el amanecer, me gustó ver el mar porque me hace pensar en qué voy a hacer y mi futuro» (Ent. 23, Comunicación personal, 15 febrero, 2017). También les otorga una atribución de independencia, les permite expresarse: «(...) así como tú eres...» (Ent. 10, Comunicación personal, 08 noviembre, 2016), sin estar sujetos a leyes o normas que los rijan.

Con respecto a la socialización yo-tú/yo-nos, la familia concentra significativamente estas relaciones, ya que los jóvenes y las jóvenes entrevistados otorgan una estima muy elevada a la convivencia con sus padres, madres, hermanas y hermanos: «me gustaría pasar más tiempo con mis hermanos, es lo que más me gustaría» (Ent. 18, Comunicación personal, 25 noviembre, 2016). Este patrón tiene su origen en un reconocimiento hacia las figuras paternas y una alta valoración de los lazos consanguíneos, derivado de una sociedad tradicional que regía a las generaciones anteriores por ideas machistas y comportamientos convencionales de autoridad masculina y de sumisión femenina (Inglehart, 1997). Por tradición, los jóvenes y las jóvenes «le deben respeto y agradecimiento» a los miembros de la familia por el esfuerzo que han hecho para que ellos puedan «salir adelante» (Inglehart \& Carballo, 2013). En esta línea, los viajes muestran el fuerte apego que tienen las personas jóvenes con la familia:

(...) creo que tenemos que disfrutar... cuando estamos con la familia... porque son las personas que siempre van a estar contigo, las que siempre te van a apoyar... entonces conocerlas más, convivir con ellos, creo que es lo más importante de los viajes...» (Ent. 28, Comunicación personal, 17 febrero, 2017).

Así también, el viaje representa una especie de «tiempo de compensación», por la falta de convivencia y dedicación de la familia nuclear o extensa con sus propios miembros, debido al estilo de vida urbano que se vive cotidianamente.

El otro núcleo de socialización es el que corresponde a la vivencia con los compañeros y las compañeras de clase, referidos mayoritariamente como interacciones yo-ellos. Identificamos que en la interacción hay una falta de identificación entre pares, ya que hay diferencias entre quienes son de Texcoco y quienes proceden de otros lugares, por sus distintos estilos de vida, gustos y cultura. Los sujetos jóvenes de Texcoco tienden a un acentuado localismo que los lleva a involucrarse fuertemente en los asuntos políticos, sociales y culturales de su contexto; sin embargo, se advierte en estos individuos la idea de que los «externos» deben alinear sus formas de pensar y actuar a los del lugar. A decir de las personas jóvenes provenientes de otros sitios, las de Texcoco muestran un gran apego a su espacio, «(...) a veces siento que están como muy... como que no salen de esta parte de Texcoco...» (Ent. 7, Comunicación personal, 04 noviembre, 2016). Algunos sujetos jóvenes locales retraen la intención de desplazarse $\mathrm{y}$, en consecuencia, de incorporar el viaje como parte de su estilo de vida: 
«siento que en cualquier lugar puedes estar bien y no necesariamente te tienes que alejar...» (Ent. 8, Comunicación personal, 04 noviembre 2016).

Los individuos estudiantes provenientes de otros sitios procuran diferenciarse de los locales, argumentando tener una personalidad más abierta, y llegando a confrontarse en algunas ocasiones: $«(. .$.$) pues yo tengo que hacer algo por ser totalmente diferente y la verdad es que a veces luchamos$ yo y mis amigos por eso...» (Ent. 7, Comunicación personal, 04 noviembre, 2016); pero en otras ocasiones ceden «(...) solo por llevar la fiesta en paz...» (Ent. 14, Comunicación personal, 14 noviembre 2016).

En los viajes que realizan los estudiantes y las estudiantes por cuestiones escolares, se manifiestan las diferencias que existen entre ellos: «(...) lo que no me gusta cuando viajo es esperar a la gente... que haya demoras, eso no me gusta, no soy nada tolerante (...)» (Ent. 17, Comunicación personal, 22 noviembre, 2016); sin embargo, el acercamiento social que genera el viaje contribuye a mejorar sus relaciones interpersonales, propiciando la comunicación y la aceptación: «(...) nos quedábamos hasta las dos o tres de la mañana platicando y pues era muy bueno el ambiente ahí (...)» (Ent. 19, Comunicación personal, 01 diciembre, 2016).

Independientemente de las diferentes personalidades de los individuos locales y regionales, las personas jóvenes asumen que los viajes forman parte de su mundo social, como formas de esparcimiento y diversión, pero además con la enfática creencia de que durante las salidas logran reforzar la convivencia.

\section{Dimensión espacial}

En esta dimensión, con respecto al espacio de vida cotidiana, identificamos ciertos elementos sobre la forma de pensar e imaginar el viaje. Por supuesto están los motivos del desplazamiento, que se pueden distinguir entre los de elección voluntaria -como los de convivencia y diversión con familiares y amigos-, que son los propiamente turísticos, y los realizados para cumplir algún compromiso de tipo educativo, religioso o deportivo. Las motivaciones dan origen a las expectativas del viaje: «(...) la verdad nunca he tenido la oportunidad de conocer la playa, sí me gustaría que hubiera un viaje a la playa para conocerla, para contemplarla (...)» (Ent. 6, Comunicación personal, 03 noviembre, 2016). En esta perspectiva, se manifiesta el anhelo por vivir una experiencia inexplorada que proyecta sus imaginarios y fantasías: «(...) me gustaría entrar al estadio de Madrid a ver un partido, (...) a mí no me gusta el fútbol casi, pero ir a ese lugar a verlo así (...) eso sería como ¡wii! lo mejor (...)» (Ent. 1, Comunicación personal, 01 noviembre, 2016). Muchos países focalizan el deseo de ser visitados por los sujetos millenials, particularmente los países europeos (9 menciones en total) como Alemania, España, Francia, Italia, Grecia, Bélgica y Holanda; le siguen China, Rusia y los Emiratos Árabes Unidos en Asia (3 menciones); Estados Unidos en América del Norte (2 menciones) y Egipto en África. Las personas jóvenes declaran el deseo de conocer otras culturas, aunque se observa que motivacionalmente existe la intención de escapar de su propio ámbito: «(...) pues sí me gustaría conocer otro tipo de cultura, no sé, tal vez algo más liberal o más donde no vean las cosas tan cuadradas como aquí, todo tiene muchos tabús (...)» (Ent. 3, Comunicación personal, 02 noviembre, 2016). Esto último deja entrever una condición de alteridad con respecto a esos «distantes lugares», cimentado en el imaginario de que son espacios de comportamientos «liberales».

Con respecto al espacio turístico, los estudiantes y las estudiantes que más han viajado han recorrido varios Estados de la República Mexicana; entre los mayormente referidos están Hidalgo, Puebla, Morelos, Michoacán, Guanajuato y Guadalajara, de la región centro del país; Yucatán, Quintana Roo y Chiapas, de la región sureste; y Sinaloa de la región noroeste, por lo que la región más visitada es la del centro, que comprende los Estados de mayor proximidad a Texcoco. A través de estos viajes, los individuos jóvenes han observado paisajes y vivido experiencias que les hacen revalorar su país, tener 
una mirada diferente sobre su entorno: «(...) Chichen-Itza me gustó demasiado, dije ¡me encanta estar aquí! Fuimos a los Cenotes, estar ahí nadando fue una experiencia única, ver su hermosura realmente es muy, muy, muy bello (...)» (Ent. 21, Comunicación personal, 13 febrero, 2017). Estas experiencias les han creado una conciencia ambiental hasta entonces no lograda en su localidad: «(...) gracias a las cosas que he visto hoy en día, pues cuido más de las cuestiones ambientales, como que trato de no tirar basura (...)» (Ent. 15, Comunicación personal, 17 noviembre, 2016).

Cabe señalar que los estudiantes y las estudiantes de la carrera de turismo son quienes mayores incentivos y oportunidades han tenido para viajar; si bien es cierto que es parte de su formación profesional, en mucho ha sido una labor impulsada por sus profesoras y profesores, quienes los han influenciado para que se dispongan a viajar, para que salgan de su zona de confort, para que experimenten sitios, personas y empleos diferentes a los que tienen en donde habitan.

De acuerdo con las entrevistas, en el uso del espacio turístico por parte de estas personas jóvenes, se manifestaron dos particularidades que trascienden del espacio cotidiano al espacio turístico: la reproducción de hábitos y la tensión sobre la seguridad. En el primer caso, independientemente de la búsqueda de nuevas emociones y experiencias, los individuos jóvenes tratan de mantener ciertos patrones de su vida cotidiana, como asistir al gimnasio o ir a la iglesia. Parecería que estos hábitos les otorgan un sentido de identidad o pertenencia que se combina con las vivencias novedosas de los viajes. En el segundo caso, la seguridad es una preocupación que los mantiene alerta en todo momento; han aprendido a ser personas precavidas en su vida cotidiana debido al nivel de riesgo que se vive en el país, en general, y en su localidad en particular: «(...) nos tenemos que cuidar hasta en el momento en el que vamos caminando o en el propio coche...» (Ent. 5, Comunicación personal, 03 noviembre, 2016), por lo que el miedo y la desconfianza son una sensación latente durante los viajes que realizan a los distintos Estados del país.

Por lo anterior, en la dimensión temporal el viaje turístico presenta su más fuerte raíz en el viaje familiar de la niñez, dotándolo de una sensación de unión y armonía. En la dimensión social, el viaje otorga a los sujetos jóvenes la posibilidad de una mayor autonomía en su forma de conducirse, y les otorga identidad con el grupo familiar, trátese de familia nuclear o extensa, así como acercamiento a sus seres contemporáneos, aún con quienes no hay una identificación. En la dimensión espacial, el individuo joven crea las expectativas de los lugares a visitar, a partir de sus imaginarios y fantasías, y al llevar a cabo la visita, es sujeto de experiencias nuevas que combina con parte de sus prácticas cotidianas.

Con los hallazgos expuestos, logramos identificar al menos dos tipos turísticos de las personas jóvenes millenials.

\section{Tipos turísticos}

Según el análisis de contenido realizado a través del modelo de Lalive, podemos caracterizar dos tipos de ethos turísticos en las personas jóvenes, denominados tradicional y liberador, según el conjunto de prácticas, creencias y valores que manifiestan sobre el viaje. Ilustramos las características distintivas de cada uno en la Tabla 2. 
Tabla 2. Tipos Turísticos de los Individuos Jóvenes Millenials en la ZMCM

\begin{tabular}{|c|c|}
\hline Ethos tradicional & Ethos liberador \\
\hline \multicolumn{2}{|c|}{ Valores fundamentales } \\
\hline $\begin{array}{l}\text { Apego a la familia. Las personas jóvenes otorgan } \\
\text { una alta valoración a las normas y modelos } \\
\text { establecidos en el grupo familiar. El respeto, } \\
\text { el agradecimiento y la obediencia hacia las } \\
\text { figuras paternas son valores que regulan su vida } \\
\text { cotidiana. Estrechar los lazos familiares es uno } \\
\text { de los motivadores de viaje más importantes para } \\
\text { aceptar el desplazamiento. }\end{array}$ & $\begin{array}{l}\text { Independencia. Las personas jóvenes disfrutan } \\
\text { la posibilidad de elegir y actuar de acuerdo a sus } \\
\text { propios impulsos y deseos, valorando el tiempo en } \\
\text { el que pueden liberarse de los convencionalismos } \\
\text { socioculturales de sus entornos cotidianos. El viaje } \\
\text { torna factibles esos momentos de libre albedrío, } \\
\text { por lo que son apreciados por sí mismos. }\end{array}$ \\
\hline $\begin{array}{l}\text { Etnocentrismo. Existe una presuposición de } \\
\text { que la cultura propia debe regir los valores y } \\
\text { comportamiento de otros. Se cree que el entorno } \\
\text { socio-cultural es adecuado y suficiente para el } \\
\text { desenvolvimiento, por lo que no hay el interés de } \\
\text { conocer directamente otros espacios. El contacto } \\
\text { con otras realidades no es buscado por sí mismo, } \\
\text { por lo que los viajes no forman parte del estilo de } \\
\text { vida. }\end{array}$ & $\begin{array}{l}\text { Cambio de paradigmas. Existe una } \\
\text { predisposición a reconocer, respetar y valorar } \\
\text { formas de pensar y vivir de maneras diferentes, } \\
\text { adaptándose a nuevos contextos y realidades, y } \\
\text { aceptando los cambios en los roles de género y } \\
\text { los patrones culturales de otras sociedades. La } \\
\text { apertura y disposición facilitan la movilidad y la } \\
\text { socialización, por lo que los viajes son aceptados } \\
\text { como parte de su estilo de vida. }\end{array}$ \\
\hline \multicolumn{2}{|c|}{ Valores expresados } \\
\hline $\begin{array}{l}\text { Entornos y hábitos conocidos. Los individuos } \\
\text { jóvenes se manifiestan más convencionales, } \\
\text { proclives a mantener tradiciones y costumbres } \\
\text { arraigadas en su comunidad, por lo que siguen los } \\
\text { hábitos seculares, particularmente las festividades } \\
\text { y ritos religiosos. Esta tendencia fomenta un } \\
\text { comportamiento con base en patrones conocidos, } \\
\text { más que en experimentar nuevos. }\end{array}$ & $\begin{array}{l}\text { Búsqueda de nuevas experiencias. El gusto por } \\
\text { tener nuevas e intensas vivencias anima a los } \\
\text { individuos jóvenes a salir de su espacio cotidiano } \\
\text { y llevar a cabo desplazamientos de largo trayecto, } \\
\text { con el propósito de tener aventuras y experiencias } \\
\text { novedosas y desafiantes. Su deseo de viajar es } \\
\text { constante. }\end{array}$ \\
\hline $\begin{array}{l}\text { Compromiso de superación. Busca prepararse } \\
\text { profesional y académicamente, bajo la creencia de } \\
\text { que sus estudios universitarios le permitirán lograr } \\
\text { un ascenso personal en la estratificación social. } \\
\text { Concibe que al incrementar su estatus, mejorará } \\
\text { su estilo de vida, y tal vez incluya a los viajes para } \\
\text { demostrar que tiene la capacidad económica para } \\
\text { llevarlos a cabo. }\end{array}$ & $\begin{array}{l}\text { Desenvoltura. La intención de saberse conducir } \\
\text { en distintas situaciones, saber cómo actuar, qué } \\
\text { decir, cómo comportarse, es una ventaja que } \\
\text { adquieren al viajar y encontrarse con personas y } \\
\text { espacios diferentes. Esto contribuye a tener un } \\
\text { estilo de vida más cosmopolita y tener confianza } \\
\text { en el desempeño de sí mismos. }\end{array}$ \\
\hline \multicolumn{2}{|c|}{ Valores contrarios } \\
\hline $\begin{array}{l}\text { Arraigoalacomunidad/Escapedelacomunidad. } \\
\text { En un sentido paradójico, identificamos que en la } \\
\text { configuración de este ethos, los sujetos jóvenes } \\
\text { presentan un arraigo a su comunidad, priorizando } \\
\text { la atención a los deberes y responsabilidades } \\
\text { que desarrollan en su vida cotidiana por sobre } \\
\text { la opción de salir en un viaje voluntario. Pero, al } \\
\text { mismo tiempo, aflora la intención de escapar de } \\
\text { las normas y convencionalismos que estructuran } \\
\text { la vida comunitaria. Así entonces, la expectativa } \\
\text { del viaje se torna en una evasión, en un recipiente } \\
\text { mental en el que se depositan fantasías y sueños } \\
\text { personales. }\end{array}$ & $\begin{array}{l}\text { Desarrollo personal/Desequilibrio personal. Se } \\
\text { concibe que los viajes son formas de aprendizaje } \\
\text { que les otorgan saberes y experiencias, } \\
\text { alimentando un amplio criterio y fomentando el } \\
\text { desarrollo de una conciencia social y ambiental } \\
\text { sobre el medio que les rodea, propiciando un } \\
\text { desarrollo integral. Sin embargo, la liberación de } \\
\text { normas y convencionalismos que favorecen los } \\
\text { viajes, también dan pauta a perder el equilibrio } \\
\text { y el autocontrol, cayendo en los excesos. El } \\
\text { viaje entonces adquiere tanto connotaciones de } \\
\text { desarrollo como de degeneración. }\end{array}$ \\
\hline
\end{tabular}




\section{Reflexiones finales}

Los tipos descritos en la caracterización de los ethos tradicional y liberador muestran dos perfiles socioculturales distintos de los individuos jóvenes de la ZMCM, en ocasiones con valores opuestos entre ellos, independientemente de su motivo de viaje. Los ethos detectados revelaron que no existe un tipo turístico uniforme según lo comúnmente descrito para el turismo de las personas jóvenes millenials en el ámbito internacional y que, efectivamente, los anclajes socioculturales confieren una identidad y una pertenencia social a determinados valores y significados que son de mayor influencia que la ejercida a través de las redes sociales y la Internet, conductores de la posmodernidad.

Es interesante identificar que los ethos mencionados encuadran en el marco explicativo de las dimensiones tradicional y de auto-expresión aportados por Inglehart (1997) para el caso de los valores en México, por lo que el ethos tradicional descrito en este texto tiene su cobertura en la dimensión del mismo nombre, y el ethos liberador en la dimensión de auto-expresión.

La aportación de la investigación que presentamos es que dichos ethos conforman patrones turísticos distintos, en los cuales el viaje no necesariamente forma parte del estilo de vida de los individuos jóvenes, habiendo un tipo de jóvenes que alberga una valoración del viaje solo bajo la perspectiva de su potencialidad para reforzar los lazos familiares y la tradición, en un contrasentido con la liberalización que naturalmente posibilitan los viajes.

Las afirmaciones hechas requieren ser corroboradas para otros casos de estudio, tanto en México como en otros países de Latinoamérica; sin embargo, el haber identificado la trascendencia de la estructura sociocultural en la conformación de los significados del viaje turístico, abre distintas posibilidades de investigación sobre los patrones de comportamiento social de los jóvenes y las jóvenes de América Latina, así como para los estudios de modernidad y posmodernidad del turismo.

\section{Lista de referencias}

Alejo, P. M., Mendoza, O. M., \& Rodríguez, H. P. (2012). Las representaciones sociales de los estudios universitarios en México. El caso de la licenciatura en turismo del Centro Universitario UAEM Texcoco. Estudios y Perspectivas en Turismo, 21(6), 1502-1520. Recuperado de: http:// www.redalyc.org/articulo.oa?id=180725030008

Barragán, M. C., López, A., Martínez, A. G., López, P., Aguilera, V. G., Salazar, J. G., \& Sánchez, B. (2014). Efecto del ayuno sobre la conducta alimentaria en estudiantes universitarios. Revista Mexicana de Trastornos Alimentarios/Mexican Journal of Eating Disorders, 5, 124-135. Recuperado de:

http://www.redalyc.org/articulo.oa?id=425741622007

Berger, P., \& Luckmann, T. (1997). Modernidad, pluralismo y crisis de sentido. Madrid: Paidós.

Bermúdez, E. (2008). Roqueros y roqueras, palitos y patitas, skaters, lesbianas y gays. El papel del consumo cultural en la construcción de representaciones de identidades juveniles (el caso de algunos grupos de jóvenes que van a los malls en Maracaibo, Venezuela). Revista Latinoamericana de Ciencias Sociales Niñez y Juventud, 6(2), 615-666. Recuperado de: http://revistaumanizales. cinde.org.co/rlcsnj/index.php/Revista-Latinoamericana/article/view/239/123.

Binkhorst, E. (2008). Turismo de co-creación, valor añadido en escenarios turísticos. Ara, Journal of Tourism Research/Revista de Investigación en Turismo, 1(1), 40-51. Recuperado de:

http://www.arajournal.net/html/ang/arajournoftouriresea11_turidecocrevaloranadienescenturis. html

Biz, A. A., Neves, A. J., \& Bettoni, E. M. (2014). O comportamento dos consumidores turísticos no uso da telefonia móvel. Caderno Virtual de Turismo, 14(1), 34-48. Recuperado de: http://www.ivt.coppe.ufrj.br/caderno/index.php/caderno/article/view/777/388

Cardoso, C. (2005). Jóvenes universitarios, ¿quiénes y cómo son? Umbral. Revista de Educación, Cultura y Sociedad. 5(9), 98-115. Recuperado de: http://sisbib.unmsm.edu.pe/BibVirtualdata/ publicaciones/umbral/v05_n09-10/a15.pdf 
Consejo Nacional de Evaluación de la Política de Desarrollo Social-Coneval. (2017). Coneval informa la evolución de la pobreza 2010-2016. Recuperado de: https://www.coneval.org.mx/SalaPrensa/ Comunicadosprensa/Documents/Comunicado-09-Medicion-pobreza-2016.pdf

Consejo Nacional de Población. (2010). La situación actual de los jóvenes en México. Recuperado de: http://www.unfpa.org.mx/publicaciones/cuadro_3.pdf

Crespi, F. (1997). Acontecimiento y estructura, por uña teoría del cambio social. Buenos Aires: Nueva Visión.

Cruz, M. S. (2000). Periferia y suelo urbano en la Zona Metropolitana de la Ciudad de México. Sociológica, 15(42), 59-90. Recuperado de: http://www.redalyc.org/html/3050/305026734004/

De Salazar, L. (2003). Los focos rojos del turismo vinculado a la cultura: cuatro casos mexicanos. Recuperado de:

http://www.equiponaya.com.ar/turismo/congreso2003/ponencias/Leticia_de_Salazar_Diaz.htm

Del Barrio, S., López, L., \& Frías, D. (2012). El tipo de incentivo como determinante en el atractivo de la promoción de venta en turismo rural. Efecto moderador del sexo, la edad y la experiencia. Revista Española de Investigación de Marketing Esic, 16(2), 103-126. Recuperado de: https:// ac.els-cdn.com/S1138144214600164/1-s2.0-S1138144214600164-main.pdf?_tid=c38f5f1cd4a5-11e7-b0d5-00000aab0f6b\&acdnat=1511919587_7343bf65f4646b0d2da180c23979714d

Dwyer, L., Edwards, D., Mistilis, N., Roman, C., Scott, N., \& Cooper, C. (2008). Megatrends Underpinning Tourism to 2020. Analysis of key drivers for change. Canberra: CRC for Sustainable Tourism Pty,Ltd.

Geifman, A. (2014). ¿Quiénes son los Millenials y qué los hace tan importantes? Recuperado de: http://www.merca20.com/que-son-los-millenials-y-por-que-hay-que-entenderlos/

Gobierno de la República de México. (2013). Plan Nacional de Desarrollo 2013-2018. Programa Sectorial de Turismo. Recuperado de: http:/www.cmic.org.mx/comisiones/Sectoriales/ turismo/2015/DOC_VIG_2015/programa_turismo.pdf

Guimarães, M. (2012). Encontros turísticos: - reflexões sobre o turismo através da subjetividade do turista. Anais Brasileiros de Estudos Turísticos, Abet, 2(1), 55-64. Recuperado de: https://abet. ufjf.emnuvens.com.br/abet/article/view/1974/1417

H. Ayuntamiento de Texcoco. (2016). Plan de Desarrollo Municipal 2016-2018. Recuperado de: http://www.texcoco.ayuntamientodigital.gob.mx/contenidos/texcoco/editor/files/PDM\%20 2016\%20OK.pdf

He, Z., Yang, L., \& Guan, W. (2014). A day-to-day route choice model based on travellers' behavioural characteristics. Procedia. Social and Behavioral Sciences, 138, 738-747. doi:10.1016/j. sbspro.2014.07.251 Recuperado de: https://ac.els-cdn.com/S1877042814041718/1-s2.0S1877042814041718-main.pdf?_tid=3afb5268-d4a6-11e7-8317-00000aab0f27\&acdnat=15119 19787 46b0eb0bfefe6de2ce8e5860c1df6e1a

Hernández, R., Fernández, C., \& Baptista, M. P. (2014). Metodología de la investigación. México, D. F.: McGraw Hill.

Hostetur. (2016). Informe sobre los hábitos y expectativas de los jóvenes. Millennials, una generación ansiosa por viajar y conocer nuevos destinos. Recuperado de: https://www.hosteltur.com/116636_ millennials-generacion-ansiosa-viajar-conocer-nuevos-destinos.html

Hu, Ch., Zhao, L., \& Huang, J. (2015). Achieving self-congruency? Examining why individuals reconstruct their virtual identity in communities of interest established within social network platforms. Computers in Human Behavior, 50, 465-475.

Inglehart, R. (1997). Modernization and postmodernization: Cultural, economic, and political change in 43 societies. New Jersey: Princenton University Press.

Inglehart, R., \& Carballo, M. (2013). ¿Existe Latinoamérica? Un análisis global de diferencias transculturales. En M. Carballo, \& A. Moreno (Coords.), El cambio de valores en América Latina. Hallazgos de la Encuesta Mundial de Valores, (pp. 17-44). México, D. F.: Centro de Estudios Sociales y de Opinión Pública, Itam. 
Instituto Nacional de Estadística y Geografía-Inegi. (2013). Cuantificando la clase media en México. Recuperado de:

http://www.inegi.org.mx/inegi/contenidos/investigacion/Experimentales/Clase_media/doc/ clase media resumen.pdf

Jacomo, $\overline{\mathrm{P}}$. (2016). Millennials: la generación de la información y la innovación. Recuperado de: https://news.sap.com/latinamerica/2016/08/12/millennials-la-generacion-de-la-informacion-yla-innovacion/

Korstanje, M. (2010). Conceptualizando: el viaje como creación cognitiva en estudiantes de turismo de Buenos Aires. Cuaderno Virtual de Turismo, 10(1), 95-111. Recuperado de: http://www. redalyc.org/html/1154/115412537008/

Kvale, S. (2011). Las entrevistas en investigación cualitativa. Madrid: Morata.

Lalive, C. (1990). Récit de vie. Ethos et comportament: pour une exégese sociologique. En J. Remy, \& R. Danielle (Dir.), Méthodes d'analyses de contenu et sociologie, (pp. 37-68). Bruxelles: Publications des Facultés Universitaires Saint Louis.

Lehmann, D. (2015). Convergencias y divergencias en la educación superior intercultural en México. Revista Mexicana de Ciencias Políticas y Sociales, 223, 133-170. Recuperado de: http://www. scielo.org.mx/pdf/rmcps/v60n223/v60n223a5.pdf

Lindón, A. (Coord.) (2000). La vida cotidiana y su espacio-temporalidad. Barcelona: Anthropos.

López, H. (2010). Ilustración de los niveles socioeconómicos en México. México, D. F.: Instituto de Investigaciones Sociales, S. C.

Lundy, L. (2015). Future traveller Tribes 2030. Building a more rewarding journey. Recuperado de: http://www.amadeus.com/documents/future-traveller-tribes-2030/amadeus-traveller-tribes2030-airline-it.pdf

Marinao, E., Torres, E., \& Chasco, C. (2014). Familiaridad con los destinos turísticos. Rol de la percepción de beneficios. Estudios Gerenciales, 30, pp. 243-251. doi:10.1016/j.estger.2014.01.019 Recuperado de: https://www.sciencedirect.com/science/article/pii/S012359231400059X

Miranda, C., \& Yanchaguano, E. (2017). Propuesta de producto turístico para los Millennials que visitan la ciudad de Guayaquil. Guayaquil: Universidad Católica de Santiago de Guayaquil. Recuperado de: http://repositorio.ucsg.edu.ec/bitstream/3317/7859/1/T-UCSG-PRE-ESPAETH-339.pdf

Monterrubio, J. C. (2013). Turismo no convencional, impactos socioculturales. México, D. F.: Trillas.

Olivares, E. (2009). México es importante destino de turismo sexual masculino: análisis. Recuperado de: http://www.jornada.unam.mx/2009/09/27/sociedad/033n1soc

Orgaz, F., \& López, T. (2015). Análisis del perfil, motivaciones y valoraciones de los turistas gastronómicos. El caso de la República Dominicana. ARA. Journal of Tourism Research, 5(1), 43-52. Recuperado de:

http://revistes.ub.edu/index.php/ara/article/view/19058/21536

Osorio, M. (2007). El Turismo en el sistema de la sociedad. En M. Osorio, \& G. Novo, (Coords.), Entorno del Turismo. Perspectivas. México, D. F.: UAEM.

Pérez, E., \& Santos, C. (2011). Diferenciación socioespacial en la Zona Metropolitana de la Ciudad de México. Investigaciones Geográficas, 74, 92-106. Recuperado de: http://www.redalyc.org/ articulo.oa? id $=56919176008$

Pérez-Rodríguez, I. L. (2012). Identidad nacional y sentidos de los jóvenes sobre su nación. Revista Latinoamericana de Ciencias Sociales, Niñez y Juventud, 10(2), 871-882. Recuperado de: http:// www.scielo.org.co/pdf/rlcs/v10n2/v10n2a07.pdf

Posso, A., \& Cardona, D. (2016). Tendencias de consumo turístico de los Millennials en la ciudad de Ibarra. Ecos de la Academia. Recuperado de: http://www.utn.edu.ec/ecos/index.php/2017/02/16/ vol-4-2016-11-i-tendencias-de-consumo-turistico-de-los-millennials-en-la-ciudad-de-ibarra/

Reporte Lobby. (2017). Millennials, la generación que sigue cambiando a la industria del turismo. Recuperado de: http://www.reportelobby.com/2017/02/millennials-generacion-turismo-reportelobby.html 
Reyes, Y., \& Osorio, M. (2015). Fe, recreación y turismo: el peregrino del pueblo de Santa María de la Asunción Tepexoyuca, Ocoyoacac, Estado de México. Revista Teoría y Praxis, 20, 69-94. Recuperado de: http://www.redalyc.org/html/4561/456149892004/

Ritzer, G. (1993). Teoría sociológica contemporánea. México, D. F.: McGraw Hill.

Rodríguez, G. (1999). Metodología de la investigación cualitativa. Málaga: Aljibe.

Sánchez, A. (2014). Los jóvenes frente al empleo y el desempleo: la necesaria construcción de soluciones multidimensionales y multifactoriales. Revista Latinoamericana de Derecho Social, 19, 133-162. Recuperado de: http://www.scielo.org.mx/pdf/rlds/n19/1870-4670-rlds-19-00133. pdf

Schütz, A. (1995). El problema de la realidad social. Buenos Aires: Amorrortu.

Schütz, A., \& Luckmann, T. (1973). Las estructuras del mundo de la vida. Buenos Aires: Amorrortu.

Sima, E. G., \& Perales, M. D. (2015). Actitudes lingüísticas hacia la maya y la elección del aprendizaje de un idioma en un sector de población joven de la ciudad de Mérida. Península, 10(1), 121-144. Recuperado de: http://www.redalyc.org/pdf/3583/358338156006.pdf

The Council of Economic Advisers. (2014). 15 Economic facts about millennials. Washington: Executive Office of the President of the United State. Recuperado de: https://obamawhitehouse. archives.gov/sites/default/files/docs/millennials_report.pdf

Unwto. (2012). La fuerza del turismo joven. Recuperado de: https://staywysetest.files.wordpress. com/2012/11/wysetc-unwto-report-spanish the-power-of-youth.pdf

Valdés, P. (2013). México, más joven y más moderno. Recuperado de: http://www.parametro.com. $\mathrm{mx} / 2013 / 03 / 507 /$

Vallejo, B., Osorio, M., Ramírez, I., Nava, G., \& Franco, S. (2013). Análisis social sobre los habitantes de la comunidad de La Peñuela, Parque Nacional Nevado de Toluca, México. Valores y comportamiento entorno al turismo. Estudios y Perspectivas en Turismo, 22, 425-449. Recuperado de: http://www.redalyc.org/articulo.oa?id=180726463004 